\title{
Qualidade de vida no transtorno obsessivo-compulsivo: uma revisão
}

\section{Quality of life in obsessive-compulsive disorder: a review}

\author{
Ricardo Cezar Torresan ${ }^{1}$, Sumaia Inaty Smaira ${ }^{2}$, Ana Teresa de Abreu Ramos-Cerqueira ${ }^{2}$, Albina Rodrigues Torres ${ }^{2}$ \\ ' Psiquiatra da Faculdade de Medicina de Botucatu da Universidade Estadual Paulista (FMB-UNESP). \\ ${ }^{2}$ Docente do Departamento de Neurologia e Psiquiatria da FMB-UNESP.
}

Recebido: 29/03/2007 - Aceito: 14/06/2007

\begin{abstract}
Resumo
Contexto: O transtorno obsessivo-compulsivo (TOC) foi considerado pela Organização Mundial da Saúde como a $10^{a}$ causa de anos vividos com incapacidade, mas pesquisas sobre qualidade de vida (QV) nesse transtorno ainda são relativamente raras. Objetivos: $\mathrm{O}$ objetivo deste trabalho foi fazer uma revisão convencional da literatura a respeito de estudos sobre qualidade de vida no TOC, publicados em português e inglês. Métodos: A busca de artigos foi feita pelos sistemas MEDLINE, PsicoInfo e LILACS, cobrindo o período de 1980 a 2007, utilizando-se como palavras-chave: qualidade de vida, transtorno obsessivo-compulsivo, prejuízo funcional e incapacidade. Resultados: Estudos populacionais apresentam indicadores indiretos de comprometimento na $\mathrm{QV}$ em pessoas com TOC, como mais desemprego, menor renda e menor índice de união conjugal estável, além de taxas relativamente altas de ideação e tentativas de suicídio. Estudos clínicos encontraram mais comprometimento na QV no TOC em comparação com algumas doenças clínicas crônicas, outros transtornos de ansiedade, quadros depressivos e mesmo esquizofrenia, em alguns aspectos. Conclusão: O impacto negativo do TOC sobre a QV de seus portadores pode ser grande, afetando vários domínios da vida e repercutindo também nos familiares. A reabilitação psicossocial deve ser vista como parte integrante essencial do tratamento e é preciso aumentar a conscientização da população sobre o problema, assim como o acesso a tratamentos adequados, para minimizar tal impacto, por vezes devastador.
\end{abstract}

Torresan, R.C. et al. / Rev. Psiq. Clín 35 (1); 13-19, 2008

Palavras-chave: Transtorno obsessivo-compulsivo, qualidade de vida, obsessões, compulsões, prejuízo funcional, incapacidade, disfunção psicossocial.

\begin{abstract}
Background: Obsessive-compulsive disorder (OCD) has been considered by the World Health Organization as the $10^{\text {th }}$ leading cause of years lived with disability, but studies on quality of life (QOL) in OCD are still scant. Objectives: A literature review of studies on quality of life in OCD. Method: A MEDLINE, Psychoinfo and LILACS literature review from 1980 to 2007 was conducted, using the following key-words: obsessive-compulsive disorder, quality of life, functional impairment and disability. Results: Population-based studies have shown indirect indicators of QOL impairment in OCD sufferers, such as more unemployment, lower income, lower number of marriages, besides considerably high rates of suicidal thoughts and attempts. Clinical studies found more QOL impairment in OCD patients compared to individuals with some chronic physical conditions, other anxiety disorders, depressive disorders and even schizophrenia, in some aspects. Conclusion: The negative impact of OCD on QOL can be severe, affecting several life domains and affecting family members as well. Psychosocial rehabilitation should be an essential part of OCD treatment and it is necessary to increase both public awareness of this disorder and the availability of appropriate treatments to minimize such impact, which can be devastating.
\end{abstract}

Torresan, R.C. et al. / Rev. Psiq. Clín 35 (1); 13-19, 2008

Key-words: Obsessive-compulsive disorder, quality of life, obsessions, compulsions, functional impairment, disability, psychosocial dysfunction.

Endereço para correspondência: Ricardo Cezar Torresan. Departamento de Neurologia e Psiquiatria da FMB-UNESP. Distrito de Rubião Jr. - 18618-970 - Botucatu, SP. E-mail: rictorresan@fmb.unesp.br 


\section{Introdução}

0 conceito de qualidade de vida em relação à saúde

Trata-se de um construto multidimensional, que reflete $o$ bem-estar físico e psíquico subjetivo dos sujeitos em diferentes domínios da vida. Tal campo de interesse envolve aspectos como bem-estar psicológico, satisfação com a vida, funcionamento social e desempenho, condições de vida e apoio social, mas não tem uma definição totalmente clara ou universalmente aceita, nem há um instrumento considerado padrão-ouro para sua avaliação. Apesar disso, desde a década de 1990 houve grande incremento nas pesquisas neste campo dentro da área da saúde, pela necessidade de se aplicar o modelo biopsicossocial das doenças na prática clínica (Katschnig, 2006).

Em se tratando de saúde mental, há importantes desafios metodológicos adicionais, pois as próprias alterações psicopatológicas podem "enviesar" ou distorcer a avaliação subjetiva de diferentes aspectos da qualidade de vida (QV), como ocorre, por exemplo, na depressão ou mania (falácia afetiva), na esquizofrenia (falácia de distorção da realidade) e nas demências ou no retardo mental (falácia cognitiva). Mesmo com todas essas dificuldades, o campo de estudos de QV em saúde, particularmente em saúde mental, vem apresentando crescente valorização na literatura, por permitir uma visão mais abrangente dos transtornos, de seu impacto na vida dos portadores e no que se refere à resposta terapêutica, que necessita ser avaliada não apenas pelos sintomas psicopatológicos (Katschnig, 2006).

\section{Peculiaridades do transtorno obsessivo-compulsivo}

O transtorno obsessivo-compulsivo (TOC) tem uma prevalência-ponto estimada em aproximadamente $1 \%$ e de $2 \%$ a $2,5 \%$ na vida, em diferentes populações (Weissman et al., 1994; Torres e Lima, 2005). Tem geralmente um quadro de início precoce, curso crônico e flutuante, e com alta comorbidade com outros transtornos ansiosos e depressivos (Torres e Smaira, 2001), além de uma grande diversidade fenomenológica, ou seja, diversos subtipos de apresentações clínicas (deMathis et al., 2006). Tratase ainda de transtorno subdiagnosticado e subtratado, muitas vezes secreto, pois vários portadores ocultam ao máximo seus sintomas e demoram muito tempo para procurar e obter tratamento adequado. Isto se deve, em grande parte, à habitual boa crítica do portador em relação aos seus sintomas, que são acompanhados de vergonha, desmoralização e muito sofrimento, justamente pela noção preservada da irracionalidade de seus pensamentos e comportamentos e da incapacidade para mudálos, além da consciência das próprias limitações (Torres e Smaira, 2001). Desconhecimento e medo podem ainda contribuir para a demora em buscar ajuda. Hollander $e t$ al. $(1996,1997)$ estimaram em 10 anos o tempo médio até o tratamento e em 17 anos a demora até a obtenção de tratamento adequado, o que, sem dúvida, prolonga o sofrimento. Além disso, sabe-se que alguns casos não melhoram substancialmente com o tratamento, mesmo que adequado, e que os efeitos colaterais dos psicofármacos também podem afetar a QV (Stein et al., 2000).

Dessa forma, seriam conseqüências comuns do TOC, apontadas por diferentes autores (Steketee $e t$ al., 1987; Hollander et al., 1996, 1997; Stein et al., 2000; Lochner et al., 2003; Moritz et al., 2005; Eisen et al., 2006): diminuição da auto-estima e do bem-estar subjetivo, interferência negativa na vida estudantil, profissional, familiar, afetiva e social, com menos conquistas acadêmicas, menores aspirações de carreira, dificuldades de relacionamento, menos amigos, menor capacidade para apreciar atividades de lazer, além dos custos indiretos para a família e para a sociedade. Em casos graves, pode haver dificuldade ou incapacidade completa para atividades diárias rotineiras e alguns portadores podem ficar totalmente presos em casa, em função de comportamentos de esquiva dos estímulos temidos.

Rapaport et al. (2005) descreveram que $26 \%$ dos pacientes com TOC apresentavam grave comprometimento da $\mathrm{QV}$, o que ocorreu em menor proporção em portadores de transtorno de pânico e fobia social, e maior nos quadros depressivos. Entre os 701 membros da Obsessive-Compulsive Foundation avaliados por Hollander et al. (1997) quanto à interferência sócio-ocupacional do TOC, 70\% relataram dificuldades na relação conjugal, $63 \%$ na socialização/ capacidade de fazer amizades, $60 \%$ na capacidade de trabalho, $59 \%$ na capacidade de estudo, $58 \%$ admitiam envolvimento de familiares nos rituais, $57 \%$ já haviam pensado em suicídio e $12 \%$ já tinham tido pelo menos uma tentativa de suicídio. Metade dos membros de uma associação de portadores de TOC da África do Sul referiu ideação suicida, e três quartos, baixa auto-estima (Stein et al., 2000). Da mesma forma, impacto relevante na vida acadêmica, ocupacional e social com níveis significativos de tendências suicidas foi relatado por pacientes da Associação Dinamarquesa de TOC (Sorensen et al., 2004).

O TOC foi apontado pela Organização Mundial da Saúde (OMS), no estudo de Sobrecarga Global das Doenças de 1999, como a décima causa de anos vividos com incapacidade em todo mundo (responsável por $2,2 \%$ da incapacitação por doenças em geral), ocupando a quinta posição entre mulheres de 15 a 44 anos, tanto em países desenvolvidos como em desenvolvimento (Murray e Lopez, 1996).

Assim, o TOC se mostra tão ou mais incapacitante que muitas doenças graves, inclusive mentais. No entanto, além de pouco estudado (Mendlowicz e Stein, 2000; Hansson, 2002; Quilty et al., 2003), o impacto negativo na QV do portador de TOC (e de seus familiares) vem sendo subestimado, talvez por este geralmente não ser considerado um transtorno mental "grave", como os quadros psicóticos crônicos ou demenciais, mas sim um transtorno "neurótico", cujas manifestações representam um continuum com a normalidade. 


\section{Objetivo e métodos}

O objetivo deste trabalho foi fazer uma revisão convencional da literatura a respeito de estudos sobre qualidade de vida no TOC, publicados em português e inglês. A busca de artigos foi feita pelos sistemas MEDLINE, PsicoInfo e LILACS, cobrindo o período de 1980 a 2007, utilizando-se como palavras-chave transtorno obsessivo-compulsivo, qualidade de vida, prejuízo funcional e incapacidade (obsessive-compulsive disorder, quality of life, functional impairment, disability). Os artigos localizados nas bases de dados e aqueles citados nas publicações avaliadas e considerados também relevantes, assim como estudos de base populacional que apresentavam dados indiretos associados à qualidade de vida, foram incluídos, totalizando 28 artigos. Foram critérios de exclusão: artigos que descreviam apenas validação de instrumentos sobre qualidade de vida e aqueles relativos a transtornos de ansiedade em geral, sem dados específicos sobre o TOC.

\section{Resultados e discussão}

Indicadores associados à qualidade de vida no TOC descritos em estudos de base populacional

Algumas variáveis sociodemográficas e de uso de serviços de saúde podem ser utilizadas como parâmetros, por se associarem frequientemente a pior qualidade de vida. Assim, apesar de não desenvolvidos especialmente para esse fim, alguns estudos realizados com amostras representativas da população geral descrevem que portadores de TOC apresentam mais freqüentemente desemprego, uso de auxílios-doença (dependência financeira), menores renda nível socioeconômico e, índice de casamento ou união conjugal estável, além de maior uso de serviços de saúde (Karno et al., 1988; Leon et al., 1995).

No levantamento britânico de morbidade psiquiátrica de 2000, indivíduos com TOC comparados a pessoas com outros transtornos "neuróticos" (ansiedade generalizada, depressão, pânico, fobias e transtorno misto depressivo-ansioso), apesar de apresentarem nível semelhante de escolaridade, estavam mais freqüentemente desempregados ou inativos, tinham menor renda, menor nível socioeconômico, menor taxa de união conjugal estável e viviam mais sozinhos (Torres et al., 2006). Referiam ainda mais interferência dos sintomas emocionais nas suas atividades diárias e sociais, pior pontuação no componente de saúde mental do SF-12 (instrumento utilizado para avaliar QV), além de apresentarem taxas significativamente mais altas de ideação (63\% vs. 38\%) e tentativas de suicídio ( $26 \%$ vs. $14 \%$ ) ao longo da vida. Ressalte-se que a prevalência de tentativas de suicídio anteriores não diferiu entre portadores de TOC com e sem outros transtornos neuróticos comórbidos, indicando que as obsessões e compulsões podem ter papel específico em relação ao risco de comportamentos suicidas. A taxa de união estável, de renda, e o nível socioeconômico e de interferência dos sintomas em atividades sociais também não diferiram nos portadores de TOC com ou sem outras comorbidades.

\section{Qualidade de vida no TOC versus na população geral}

Diversos estudos mostram que, como esperado, a QV no TOC é bem pior em todos os domínios, quando comparada aos resultados obtidos na população geral (Bobes et al., 2001; Rapaport et al., 2005; Stengler-Wenzke et al., 2006; Eisen et al., 2006, Rodriguez-Salgado et al., 2006). No entanto, estudo de Koran et al. (1996) com 60 pacientes ambulatoriais que apresentavam sintomas moderados ou graves não encontrou diferenças em relação a taxa de casamento, abuso de álcool e tentativas de suicídio, índices indiretos de $\mathrm{QV}$, mas pior desempenho de papéis sociais e funcionamento social, mais desempregados e solteiros.

Segundo estudo de Eisen et al. (2006), 34\% dos 197 adultos com TOC eram incapazes de trabalhar fora, $5 \%$ eram incapazes para qualquer atividade doméstica e 14\% estavam recebendo auxílio-doença. Os resultados que mais diferenciaram os pacientes dos sujeitos de comparação da população geral foram observados nas dimensões de saúde mental, limitação de papéis por problemas emocionais e vitalidade. Rodriguez-Salgado et al. (2006) encontraram diferenças significativas, para pior, no TOC em relação à população geral espanhola em todos os domínios, com exceção de saúde física e dor.

\section{Qualidade de vida em portadores de TOC versus portadores de doenças clínicas}

Alguns trabalhos investigaram comparativamente QV no TOC em relação a algumas doenças clínicas crônicas. Assim, Koran et al. (1996) descreveram em pessoas com TOC pior QV do que naquelas com diabetes grave, e Bobes et al. (2001) encontraram pior QV entre portadores de TOC do que em pacientes com insuficiência renal crônica em hemodiálise ou já transplantados.

Qualidade de vida no TOC versus outros

transtornos de ansiedade

Um trabalho conduzido nos Estados Unidos em 1987 por Steketee et al. comparou a QV no TOC em relação a pessoas com fobias e transtorno de ansiedade generalizada, encontrando pior nível socioeconômico, mais desemprego, depressão e sentimentos de raiva, inutilidade e inadequação, além de mais desconforto interpessoal e baixa auto-estima. Os autores consideram que o início precoce dos sintomas pode interferir no desenvolvimento de habilidades interpessoais.

No Canadá, Quilty et al. (2003) encontraram no TOC comprometimento da QV comparável ao transtorno de pânico e à fobia social, enquanto Antony et al. (1998) não encontraram diferença na pontuação global na Illness 
Intrusiveness Rating Scale, também em relação a pacientes com pânico ou fobia social, mas sim uma interferência ou "intrusividade" maior dos sintomas do TOC em atividades passivas que exigem concentração, como leitura (por necessidade de repetir ou contar, medo de certas palavras etc.), e de expressão de religiosidade (em função de obsessões religiosas e compulsões envolvendo orações).

\section{Qualidade de vida no TOC versus esquizofrenia}

Estudo conduzido na Índia, em 2002, por Jayakumar et al. encontrou melhor QV em todos os domínios no TOC, incluindo melhor renda e desempenho sócio-ocupacional, porém semelhante no que se referia ao papel conjugal/sexual. Apesar de o nível de desemprego ter sido menor entre portadores de TOC, muitos não trabalhavam fora, eram estudantes ou donas de casa. Segundo os autores, problemas na esfera sexual no TOC podem estar relacionados ao medo de sujeira e contaminação e merecem abordagens terapêuticas específicas.

Para Calvocoressi et al. (1998), apesar de indivíduos com TOC terem tido melhor pontuação geral, não diferiram dos portadores de esquizofrenia em relação ao desempenho no trabalho e às habilidades da vida diária, sendo ambos os grupos piores nesses aspectos do que portadores de depressão. Portadores de TOC e de esquizofrenia não diferiram em relação a tempo de trabalho perdido, fontes de renda e qualidade das amizades. $\mathrm{O}$ estudo de Bobes et al. (2001), realizado na Espanha, descreveu QV subjetiva tão ruim entre portadores de TOC quanto entre portadores de esquizofrenia em tratamento ambulatorial, que se assemelharam em medidas globais de saúde mental. É de se notar que, enquanto os portadores de esquizofrenia foram considerados pelos clínicos como tendo maior prejuízo nas áreas relativas à saúde física, os portadores de TOC tiveram pior avaliação em aspectos sociais e ocupacionais.

Pacientes com esquizofrenia, comparados àqueles com TOC grave por Bystritsky et al. (2001), nos Estados Unidos, apresentaram prejuízo social considerável e equivalente pré-tratamento, mas, com o tratamento, houve maior melhora funcional no grupo com TOC, ainda assim melhora considerada insatisfatória, principalmente no que se referia a desempenho social e capacidade de vida independente. Para os autores, a boa crítica dos portadores de TOC, que em geral têm melhor noção das próprias insuficiências e se sentem impotentes para controlar os sintomas, geraria tal comprometimento do bem-estar subjetivo, sentimentos de desmoralização e sintomas depressivos. É interessante assinalar que um trabalho que avaliou a capacidade crítica de pacientes psicóticos e aspectos de QV (Hasson-Ohayon et al., 2006) encontrou correlação inversa entre o grau de insight e QV, ou seja, melhor crítica associou-se com redução do bem-estar emocional, pior avaliação do status vocacional e menor grau de satisfação econômica. Isso pode explicar o fato de alguns portadores de TOC apre- sentarem mais sofrimento e pior percepção subjetiva de $\mathrm{QV}$ do que pessoas com transtornos mentais que envolvem maior comprometimento da crítica.

Estudo recente realizado na Alemanha (StenglerWenzke et al., 2006) encontrou, em pacientes com TOC e sem comorbidades associadas, pior avaliação do relacionamento social (que envolve satisfação com relacionamentos sociais, com apoio de amigos e sexualidade), bem-estar psicológico (tristeza, ansiedade, insatisfação consigo mesmo) e QV em geral, possivelmente em função de sentimentos de desmoralização e impotência ("É como estar louco, mas com a mente consciente"). Para os autores, as características clínicas da esquizofrenia levariam à adaptação gradual, com menores aspirações pessoais e profissionais, modificando a percepção subjetiva da QV.

Qualidade de vida no TOC versus

outros transtornos mentais

Bobes et al. (2001) estudaram 36 pacientes com TOC na Espanha, sendo 61\% considerados casos graves, e obtiveram indicadores de pior QV, quando comparados a pacientes deprimidos ou dependentes de heroína, havendo impacto negativo principalmente na carreira $\mathrm{e}$ nos relacionamentos com amigos e familiares. Como já mencionado, o desempenho no trabalho, as habilidades para a vida diária e a pontuação geral no ano anterior foram piores em pessoas com TOC do que naquelas com depressão, pelo estudo de Calvocoressi et al. (1998).

\section{Impacto da gravidade do TOC e dos tipos de sintoma na qualidade de vida}

O TOC é um quadro que apresenta ampla variação na gravidade de suas manifestações clínicas, que vão desde casos leves até aqueles extremamente graves. Koran $e t$ al. (1996) descreveram que, quanto maior a gravidade, pior o funcionamento social dos portadores, independentemente da presença de depressão associada. Para Stein et al. (2000), apesar de haver alguma correlação entre maior gravidade dos sintomas e pior $\mathrm{QV}$, esta não é direta, já que alguns pacientes, mesmo com sintomatologia importante, conseguem manter os papéis sociais e cumprir suas obrigações a contento. Isto talvez se deva à especificidade idiossincrática dos temores obsessivos e à capacidade que alguns portadores têm de manter seus rituais restritos à sua casa ou seu quarto, controlando-os em situações públicas.

Para Masellis et al. (2003) e Eisen et al. (2006), a gravidade das obsessões e dos sintomas depressivos associados é determinante de uma pior $\mathrm{QV}$, comprometendo principalmente o bem-estar subjetivo, as relações sociais e a capacidade para o lazer, enquanto a gravidade das compulsões estaria mais especificamente relacionada à capacidade de trabalhar fora (Eisen et al., 2006). Os pensamentos obsessivos interfeririam consideravelmente em atividades intencionais como ler, escrever, contar, 
ou simplesmente manter a concentração (Masellis et al., 2003).

No estudo de Eisen et al. (2006), pacientes com pontuação acima de 20 na Escala de Yale-Brown - que tem pontuação máxima de 40 - (Goodman et al., 1989) tiveram comprometimento mais marcado da $\mathrm{QV}$ em relação aos demais, e os autores chegam a sugerir esse ponto de corte para se considerar o diagnóstico de TOC na próxima (quinta) revisão do DSM. Ressalte-se que houve também correlação estatisticamente significante - porém de menor magnitude que a gravidade dos sintomas obsessivo-compulsivos (SOC) - entre a pontuação na escala que mede o grau de crítica ou insight (Brown Assessment of Beliefs Scale) e cinco dos sete instrumentos de mensuração da QV.

Rodriguez-Salgado et al. (2006) estudaram 64 indivíduos com TOC e também encontraram correlação entre gravidade do transtorno e todas as subescalas da SF-36, assim como com a pontuação na escala de depressão de Hamilton. Porém, o impacto negativo na QV não pode ser atribuído aos sintomas afetivos, pois a média na escala de depressão foi baixa. Enquanto a subescala de obsessões correlacionou-se com todos os domínios da SF-36, a subescala de compulsões correlacionou-se apenas com funcionamento social, papel emocional, saúde mental e vitalidade. Fatores sociodemográficos, idade de início e tempo de evolução dos SOC não afetaram significativamente a percepção de QV.

Apesar de a maioria dos estudos apontar para a associação esperada entre gravidade dos sintomas e comprometimento da $\mathrm{QV}$, é importante ressaltar que mesmo quadros subclínicos de transtornos ansiosos podem interferir na $\mathrm{QV}$ e no funcionamento social dos portadores (Mendlowicz e Stein, 2000).

\section{Efeito do tratamento do TOC na qualidade de vida}

Um aspecto importante a ser considerado é o impacto do tratamento na $\mathrm{QV}$ de pessoas com TOC. Moritz et al. (2005) descreveram comprometimento importante do bem-estar subjetivo, vitalidade e funcionamento social em 79 pacientes avaliados, havendo melhora significativa da QV em respondedores, quando comparados a não respondedores, mas em geral persistindo comprometimento após o tratamento. O número de $\mathrm{SOC}$ e a gravidade da depressão foram melhores preditores da manutenção do comprometimento da $\mathrm{QV}$ do que a gravidade dos SOC em geral. $\mathrm{O}$ bem-estar físico esteve mais comprometido em pacientes com rituais de limpeza e lavagem.

Entre 30 portadores de TOC grave e resistente a abordagens terapêuticas convencionais que foram internados em hospital-dia para tratamento, houve melhora em apenas três de oito aspectos de QV até a alta, e não houve correlação direta com a gravidade (Bystritsky $e t$ $a l ., 1999)$. As maiores mudanças ocorreram em satisfação subjetiva com atividades, saúde e vida em geral. Hollander et al. (1997) descreveram, na maioria dos casos tratados, apenas melhora pequena em relação a emprego, carreira e socialização com o tratamento.

Tenney et al. (2003) estudaram 101 pacientes com TOC que foram tratados com paroxetina ou venlafaxina por 12 semanas, obtendo melhora semelhante em todas as subescalas do instrumento de avaliação (Lancashire Quality of Life Profile), independentemente da melhora sintomática. Para esses autores, pode ocorrer melhora na $\mathrm{QV}$, em curto prazo, mesmo entre os que não tenham respondido ao tratamento, por um efeito inespecífico deste, talvez por se sentirem mais esperançosos.

De todo modo, parece não existir associação linear entre melhora dos sintomas e da $\mathrm{QV}$, havendo necessidade de se programar estratégias ativas de reabilitação, que favoreçam também a melhora funcional de pessoas com TOC (Bystritsky et al., 2001). Na verdade, muitos portadores de quadros mais graves passam anos profundamente limitados por seus sintomas, praticamente isolados em casa, de modo que "ir em busca do tempo perdido" ou recuperar os projetos de vida deixados pelo caminho em função do transtorno em geral não é tarefa fácil ou automática, devendo envolver profissionais (por exemplo, terapeutas ocupacionais, psicólogos) que trabalhem especificamente a questão da reinserção social dessas pessoas.

\section{Impacto familiar do TOC}

Sem dúvida, o impacto negativo do TOC nos familiares é muito mais do que econômico, envolvendo acomodação aos rituais e às solicitações bizarras do paciente, mudanças de rotina, impossibilidade de receber visitas, de ter atividades de lazer, pela dependência do paciente em relação a seus cuidadores primários. É comum, em casos mais graves, o paciente ficar incapacitado até para realizar as atividades mais simples do dia-a-dia, como tarefas domésticas ou higiene pessoal. Diferentemente de famílias que convivem com pacientes psicóticos ou usuários de drogas, por exemplo, no TOC há uma previsibilidade dos comportamentos e solicitações do portador, de tal forma que gradualmente a família vai se adaptando aos mesmos comportamentos de esquiva passiva ou ativa (rituais compulsivos), por vezes sem se dar conta do comprometimento que isso gera na sua própria QV. Compaixão, vergonha, raiva e muita ambivalência são sentimentos comuns entre os familiares, cuja sobrecarga emocional pode ter efeitos deletérios na sua própria saúde mental (Stengler-Wenzke et al., 2004).

\section{Conclusão}

Apesar do número relativamente pequeno de estudos encontrados, com diferentes amostras e utilizando instrumentos de avaliação diversos (Quadro 1), esta revisão da literatura indica que o impacto do TOC na QV pode ser devastador, afetando negativamente vários domínios da vida do portador (acadêmico, ocupacional, social e familiar) 
e repercutindo também nos familiares e amigos. $O$ custo social também pode ser alto, em função do desemprego, da dependência financeira da família, do pagamento de auxílios-doença e do uso de serviços de saúde, em geral por adultos jovens, que se encontram em uma das fases potencialmente mais produtivas de suas vidas (Stein $e t a l$., 2000). O risco de suicídio provavelmente também vem sendo subestimado, merecendo mais atenção por parte de profissionais de saúde mental e gestores de saúde (Hollander et al., 1997; Stein et al., 2000; Torres et al., 2006).

Quadro 1. Síntese dos principais estudos clínicos que avaliaram QV no TOC (estudos populacionais e de revisão não incluídos)

\begin{tabular}{|c|c|c|c|c|}
\hline $\begin{array}{l}\text { Autor, ano de } \\
\text { publicação e país }\end{array}$ & $\begin{array}{l}\text { N TOC } \\
\text { (faixa etária) }\end{array}$ & $\begin{array}{l}\text { Instrumento(s) } \\
\text { de QV }\end{array}$ & Grupo(s) de comparação (N) & Principais resultados/conclusões \\
\hline $\begin{array}{l}\text { Steketee et al., } \\
1987 \text { (EUA) }\end{array}$ & 75 & Não estruturado & $\begin{array}{l}\text { Fóbicos sociais (13) } \\
\text { Fóbicos simples (18) } \\
\text { Agorafóbicos (23) } \\
\text { Tr. ansiedade generalizada (11) }\end{array}$ & $\begin{array}{l}\text { TOC: mais desemprego, pobreza, } \\
\text { sentimentos de raiva, inutilidade e } \\
\text { inadequação, baixa auto-estima }\end{array}$ \\
\hline $\begin{array}{l}\text { Antonyet al., } 1998 \\
\text { (Canadá) }\end{array}$ & 51 (18-65 anos) & $\operatorname{IIRS}(1)$ & $\begin{array}{l}\text { Tr. pânico (35) e } \\
\text { Fobia social (49) }\end{array}$ & $\begin{array}{l}\text { Prejuízo funcional semelhante } \\
\text { nos três grupos }\end{array}$ \\
\hline $\begin{array}{l}\text { Calvocoressi et } \\
\text { al., } 1998 \text { (EUA) }\end{array}$ & 17 & $\mathrm{GAF}^{(2)}$ & $\begin{array}{l}\text { Depressão maior (17) e } \\
\text { esquizofrenia (17) }\end{array}$ & $\begin{array}{l}\text { TOC: prejuízo funcional maior que } \\
\text { depressivos e semelhante aos } \\
\text { portadores de esquizofrenia }\end{array}$ \\
\hline $\begin{array}{l}\text { Bystritskyet al., } \\
1999 \text { (EUA) }\end{array}$ & 30 (18-56 anos) & $\begin{array}{l}\text { Lehman's QOL } \\
\text { Scale }^{(3)}\end{array}$ & $\begin{array}{l}\text { Comparou pacientes com TOC } \\
\text { antes e depois do tratamento }\end{array}$ & $\begin{array}{l}\text { Melhora na pontuação da YBOCS } \\
\text { não associada a modificações nos } \\
\text { escores de QV }\end{array}$ \\
\hline $\begin{array}{l}\text { Bobes et al., } 2001 \\
\text { (Espanha) }\end{array}$ & 36 (13-85 anos) & $\begin{array}{l}\text { WHO-DAS } \\
\text { SF-36 }\end{array}$ & $\begin{array}{l}\text { População geral } \\
\text { Pacientes com TOC americanos } \\
\text { Portadores de esquizofrenia (362) } \\
\text { Deprimidos (729) } \\
\text { Dependentes de heroína } \\
\text { Pacientes renais crônicos graves }\end{array}$ & $\begin{array}{l}\text { Pior QV no TOC, exceto quando } \\
\text { comparado aos portadores de } \\
\text { esquizofrenia }\end{array}$ \\
\hline $\begin{array}{l}\text { Bystritsky et al., } \\
2001 \text { (EUA) }\end{array}$ & 31 & $\begin{array}{l}\text { Lehman's OOL } \\
\text { Scale }{ }^{(3)} \mathrm{e} \\
\text { ILSS }^{(6)}\end{array}$ & Portadores de esquizofrenia (68) & $\begin{array}{l}\text { Prejuízo social considerável e } \\
\text { equivalente nos dois grupos pré- } \\
\text { tratamento, porém maior melhora } \\
\text { funcional no TOC }\end{array}$ \\
\hline $\begin{array}{l}\text { Maselliset al., } \\
2003 \text { (Canadá) }\end{array}$ & 43 (18-65 anos) & IIRS $(1)$ & - & $\begin{array}{l}\text { Depressão comórbida e gravidade } \\
\text { das obsessões tiveram maior } \\
\text { impacto na QV }\end{array}$ \\
\hline $\begin{array}{l}\text { Quilty et al., } 2003 \\
\text { (Canadá) }\end{array}$ & 65 (15-68 anos) & $\begin{array}{l}\operatorname{SDS}^{(7)}, \operatorname{MOS}^{(8)} \\
\operatorname{SAS}^{(9)}, \operatorname{LSRDS}^{(10)} \\
\operatorname{LCRDP}^{(11)}\end{array}$ & $\begin{array}{l}\text { Tr. pânico (181) e } \\
\text { Fobia social (91) }\end{array}$ & $\begin{array}{l}\text { Impacto semelhante na QV dos } \\
\text { indivíduos dos três grupos }\end{array}$ \\
\hline $\begin{array}{l}\text { Tenney et al., } 2003 \\
\text { (Holanda) }\end{array}$ & 150 (18-70 anos) & LqoLP(12) & - & $\begin{array}{l}\text { Melhora da QV não relacionada à } \\
\text { melhora dos sintomas OC }\end{array}$ \\
\hline $\begin{array}{l}\text { Moritz et al., } 2005 \\
\text { (Alemanha) }\end{array}$ & 79 & SF-36 $6^{(5)}$ & População geral (2.914) & $\begin{array}{l}\text { Gravidade da depressão } \\
\text { comórbida e número de sintomas } \\
\text { OC tiveram maior impacto na QV }\end{array}$ \\
\hline $\begin{array}{l}\text { Eisen et al., } 2006 \\
\text { (EUA) }\end{array}$ & 197 (> 18 anos) & $\begin{array}{l}\text { Q-LES-Q }{ }^{(13)} \mathrm{e} \\
\text { SF- } 36^{(5)}\end{array}$ & - & $\begin{array}{l}\text { Todos os aspectos de QV afetados } \\
\text { pelo TOC; associação com a } \\
\text { gravidade do TOC e depressão } \\
\text { comórbida }\end{array}$ \\
\hline $\begin{array}{l}\text { Rodriguez- } \\
\text { Salgadoet al., } \\
2006 \text { (Espanha) }\end{array}$ & 64 & SF-36 $6^{(5)}$ & População geral & $\begin{array}{l}\text { Comorbidade clínica e } \\
\text { psiquiátrica, gravidade do TOC e } \\
\text { desemprego: maior impacto na QV }\end{array}$ \\
\hline $\begin{array}{l}\text { Stengler-Wenzke } \\
\text { et al., } 2006 \\
\text { (Alemanha) }\end{array}$ & 75 (21-72 anos) & WHOQOL-BREF(4) & $\begin{array}{l}\text { População geral e } \\
\text { portadores de esquizofrenia }\end{array}$ & $\begin{array}{l}\text { Aspectos de bem-estar } \\
\text { psicológico e relacionamentos } \\
\text { sociais piores no TOC do que na } \\
\text { esquizofrenia }\end{array}$ \\
\hline
\end{tabular}

(1)IIIness Intrusiveness Rating Scale; ${ }^{(2)}$ Global Assessment of Functioning; ${ }^{(3)}$ Lehman's Quality of Life Scale; ${ }^{(4)}$ World Health Organization Short Disability Assessment Schedule; ${ }^{(5)}$ Medical Outcomes Survey 36-Item Short Form Health Survey; ${ }^{\left({ }^{6}\right)}$ Independent Living Skills Survey; ${ }^{(7)}$ Sheehan Disability Scale; ${ }^{(8)}$ Medical Outcomes Study Health Survey; ${ }^{(9)}$ Social Adjustment Scale-Self Report; ${ }^{(10)}$ Liebowitz Self-Rating Disability Scale; ${ }^{(11)}$ Liebowitz Clinician-Rated Disability Profile; ${ }^{(12)}$ Lancashire Quality of Life Profile; ${ }^{(13)}$ Quality of Life Enjoyment 
Mais estudos sistemáticos são necessários nessa área, inclusive para se avaliar se diferentes dimensões de sintomas ou subtipos do TOC têm algum impacto diferencial na $\mathrm{QV}$ dos portadores, uma vez que se trata de um quadro extremamente heterogêneo (deMathis et al., 2006).

A reabilitação psicossocial, com treinamento de habilidades específicas, deve ser considerada parte integrante essencial do planejamento terapêutico para pacientes com TOC, uma vez que a melhora sintomatológica não se reflete direta ou necessariamente em melhora funcional ou da $\mathrm{QV}$, podendo haver déficits psicossociais residuais relevantes (Bystritsky et al., 2001; Stengler-Wenzke et al., 2006).

Por fim, para minimizar o sofrimento e o impacto na $\mathrm{QV}$ de portadores de TOC e familiares, é preciso melhorar a conscientização da população geral sobre o problema, assim como possibilitar o rápido acesso a tratamentos adequados. Isto implica mais treinamento e atualização para profissionais de saúde, inclusive em serviços de atenção primária, para que se melhore a detecção do TOC, que infelizmente ainda é um transtorno oculto e insatisfatoriamente tratado.

\section{Referências}

Antony, M.M.; Roth, D.; Swinson, R.P.; Huta, V.; Devins, G.M. - Illness intrusiveness in individuals with panic disorder, obsessive-compulsive disorder, or social phobia. J Nerv Ment Dis 186: 311-315, 1998.

Bobes, J.; González, M.P.; Bascarán, M.T.; Arango, C.; Saiz, P.A.; Bousono, M.I. - Quality of life and disability in patients with obsessive-compulsive disorder. Eur Psychiatry 16: 239-245, 2001.

Bystritsky, A.; Saxena, S.; Maidment, K.; Vapnik, T.; Tarlow, G.; Rosen, R. - Quality-of-life changes among patients with obsessive-compulsive disorder in a partial hospitalization program. Psychiatr Serv 50(3): 412-414, 1999.

Bystritsky, A.; Liberman, R.P.; Hwang, S.; Wallace, C.J.; Vapnik, T.; Maindment, $K$, et al. - Social functioning and quality of life comparisons between obsessive-compulsive and schizophrenic disorders. Depress Anxiety 14: 214-218, 2001.

Calvocoressi, L.; Libman, D.; Vegso, S.J.; McDougle, C.J.; Price, L.H. - Global functioning of impatients with obsessive-compulsive disorder, schizophrenia, and major depression. Psychiatr Serv 49(3): 379-338, 1998.

deMathis, M.A.; Diniz, J.B.; Rosário, M.C.; Torres, A.R.; Hoexter, M.; Hasler, G., et al. - What is the optimal way to subdivide obsessive-compulsive disorder? CNS Spectr 11(10): 762-779, 2006.

Eisen, J.L.; Mancebo, M.A.; Pinto, A.; Coles, M.E.; Pagano, M.E.; Stout. R., et al. - Impact of obsessive-compulsive disorder on quality of life. Compr Psychiatry 47: 270-275, 2006.

Goodman, W.K.; Price, L.H.; Rasmussen, S.A.; Mazure, C.; Fleischmann. R.L.; Hill, C.L., et al. - The Yale-Brown Obsessive-Compulsive Scale I. Development, use and reliability. Arch Gen Psychiatry 46: 1006-1011, 1989.

Hansson, L. - Quality of life in depression and anxiety. Int Rev Psychiatry 14: 185-189, 2002.

Hasson-Ohayon, I.; Kravetz, S.; Roe, D.; David, A.S.; Weiser, M. - Insight into psychosis and quality of life. Compr Psychiatry 47: 265-269, 2006.

Hollander, E.; Know, J.H.; Stein, D.J.; Broatch, J.; Rowland, T.; Himelein, C.A. - Obsessive-compulsive and spectrum disorders: overview and quality of life issues. J Clin Psychiatry 57(supl 8): 3-6, 1996.

Hollander, E.; Stein, D.; Know, J.H.; Rowland, C.; Wong, C.M.; Broatch, J. Psychossocial function and economic costs of obsessive-compulsive disorder. CNS Spectrums 2: 16, 1997.
Jayakumar, C.; Jagadheesan, K.; Verma, A.N. - Disability in obsessivecompulsive disorder: a comparison with schizophrenia. Int J Rehabil Res 25: 147-151, 2002.

Karno, M.; Golding, J.M.; Sorenson, S.B.; Burnam, A. - The epidemiology of obsessive-compulsive disorder in five US communities. Arch Gen Psychiatry 45: 1094-1109, 1988.

Katschnig, H. - Quality of life in mental disorders: challenges for research and clinical practice. World Psychiatry 5(3): 139-145, 2006.

Koran, L.M.; Thienemann, M.L.; Davenport, R. Quality of life for patients with obsessive-compulsive disorder. Am J Psychiatry 153: 783-788, 1996.

Koran, L.M. - Quality of life in obsessive-compulsive disorder. Psychitr Clin North Am 23: 509-517, 2000

Leon, A.C.; Portera, L.; Weissman, M.M. - The social costs of anxiety disorders. Br J Psychiatry 166 (supl 27): 19-22, 1995.

Lochner, C.; Mogotsi, M.; du Toit, P.L.; Kaminer, D.; Niehaus, D.J.; Stein, D.J. - Quality of life in anxiety disorders: a comparison of obsessivecompulsive disorder, social anxiety disorder, and panic disorder. Psychopathol 36: 255-262, 2003.

Masellis, M.; Rector, N.A.; Richter, M.A. - Quality of life in OCD: differential impact of obsessions, compulsions, and depression comorbidity. Can J Psychiatry 48 (2): 72- 77, 2003.

Mendlowicz, M.V.; Stein, M.D. - Quality of life in individuals with anxiety disorders. Am J Psychiatry 157: 669-682, 2000.

Moritz, S.; Rufer, M.; Fricke, S.; Karow, A.; Morfeld, M.; Jelinek, L., et al. - Quality of life in obsessive-compulsive disorder before and after treatment. Compr Psychiatry 46: 453-454, 2005.

Murray, C.J.L.; Lopez, A.D. - The global burden of disease. Harvard University Press, Boston (MA), 1996.

Quilty, L.C.; Van Ameringen, M.; Mancini, C.; Oakman, J.; Farvolden, P. - Quality of life and the anxiety disorders. J Anxiety Disord 17: 405-426, 2003.

Rapaport, M.H.; Clary, C.; Fayyad, R.; Endicott, J. - Quality-of-life impairment in depressive and anxiety disorders. Am J Psychiatry 162: 1171-1178, 2005.

Rodriguez-Salgado, B.; Dolengevich-Segal, H.; Arrojo-Romero, M.; CastelliCandi, P.; Navio-Acosta, M.; Perez-Rodrigues, M.M., et al. - Perceived quality of life in obsessive-compulsive disorder: related factors. BMC Psychiatry 6: 20, 2006.

Sorensen, C.B.; Kirkeby, L.; Thomsen, P.H. - Quality of life with OCD: a selfreported survey among members of the Danish OCD Association. Nord J Psychiarty 58(3): 231-236, 2004.

Stein, D.J.; Allen, A.; Bobes, J.; Eisen, J.L.; Figuera, M.L.; Koran, L.M., et al. - Quality of life in obsessive-compulsive disorder. CNS Spectr $5(6)$ [supl 4]: 37-39, 2000.

Steketee, G.; Grayson, J.B.; Foa, E.B. - A comparison of characteristics of obsessive-compulsive disorder and other anxiety disorders. J Anxiety Disord 1: 325-335, 1987.

Stengler-Wenzke, K.; Trosbach, J.; Dietrich, S.; Angermeyer, M.C. - Coping strategies used by the relatives of people with obsessive-compulsive disorder. J Adv Nurs 48(1): 35-42, 2004.

Stengler-Wenzke, K.; Kroll, M.; Matschinger, H.; Argermeyer, M.C. - Subjective quality of life of patients with obsessive-compulsive disorder. Soc Psychiatry Psychiatr Epidemiol 1-7, 2006.

Tenney, N.H.; Denys, D.A.J.P.; van Megen, H.J.G.M.; Glas, G.; Westenberg, H.G.M. - Effect of a pharmacological intervention on quality of life in patients with obsessive-compulsive disorder. Int Clin Psychopharmacol 18(1): 29-33, 2003.

Torres, A.R.; Smaira, S.I. - Quadro clínico do transtorno obsessivo-compulsivo. Rev Bras Psiquiatria 23(supl II): 6-9, 2001.

Torres, A.R.; Lima, M.C.P. - Epidemiologia do transtorno obsessivo-compulsivo: uma revisão. Rev Bras Psiquiatria 27(3): 237-242, 2005.

Torres, A.R.; Prince, M.J.; Bebbington, P.E.; Bhugra, D.; Brugha, T.S.; Farrell, M., et al. - Obsessive-compulsive disorder: prevalence, comorbidity, impact, and help-seeking in the British National Psychiatric Morbidity Survey of 2000. Am J Psychiatry 163(11): 1978-1985, 2006.

Weissman, M.M.; Bland, R.C.; Canino, G.J.; Greenwald, S.; Hwu, H.; Lee, C.K., et al. - The cross national epidemiology of obsessive compulsive disorder. J Clin Psychiatry 55(supl 3): 5-10, 1994. 